Article

\title{
Avaliação de Métodos de Estimativa da Evapotranspiração de Referência (ETo) Diária Para Regiões dos Estados do Rio de Janeiro e Espírito Santo
}

\author{
Eluã Ramos Coutinho ${ }^{1}$ (D) Jonni Guiller Ferreira Madeira ${ }^{2}$, Robson Mariano da Silva ${ }^{3}$, \\ Elizabeth Mendes de Oliveira ${ }^{2}$, Angel Ramon Sanchez Delgado ${ }^{3}$ \\ ${ }^{1}$ Programa de Pós-Graduação em Ciência, Tecnologia e Inovação em Agropecuária, \\ Universidade Federal Rural do Rio de Janeiro, Seropédica, RJ, Brasil. \\ ${ }^{2}$ Grupo de Nanociência e meio ambiente, Departamento de Engenharia Mecânica, \\ Centro Federal de Educação Tecnológica Celso Suckow da Fonseca, Angra dos Reis, RJ, Brasil. \\ ${ }^{3}$ Departamento de Matemática, Universidade Federal Rural do Rio de Janeiro, \\ Seropédica, RJ, Brasil.
}

Recebido em: 21 de Junho de 2019 - Aceito em: 7 de Maio de 2020

\begin{abstract}
Resumo
O alto consumo de água pela agricultura torna cada vez mais essencial o conhecimento da evapotranspiração de referência $(E T o)$ para a realização do manejo da irrigação. Entretanto, definir um método adequado às diferentes localidades está associado à disponibilidade dos dados meteorológicos e a adaptação dos métodos às localidades aplicadas. Assim, o objetivo deste trabalho foi comparar e avaliar o desempenho dos métodos de ETo: Hargreaves-Samani, Jensen-Haise, Benavides \& Lopez e Hamon com o método padrão Penman Monteith FAO-56, para estimar a ETo diária de seis regiões, três do estado do Rio de Janeiro e três do estado do Espírito Santo. As variáveis meteorológicas empregadas foram cedidas pelo Centro de Previsão de Tempo e Estudos Climáticos e do Instituto Nacional de Pesquisas Espaciais (CPTEC - INPE). O desempenho dos modelos foi avaliado por diferentes técnicas estatísticas onde o modelo que melhor se adaptou às localidades estudadas nos dois estados foi o de Jensen Haise, tendo obtido os índices de correlação (r) entre 0,73 a 0,94 e a confiabilidade (C) entre 0,60 a 0,90 com o modelo de Penman Monteith.
\end{abstract}

Palavras-chave: irrigação, dados meteorológicos, Penman Monteith.

\section{Evaluation of Methods of Estimation of Evapotranspiration of Reference (ETo) Daily for Regions of the States of Rio De Janeiro and Espírito Santo}

\begin{abstract}
The high consumption of water by agriculture makes knowledge about reference evapotranspiration (ETo) for irrigation management more and more essential. However, define an appropriate method to the different locations is associated with the availability of meteorological data and the adaptation of the methods to the application sites. Thus, the objective of this study is to compare and evaluate the performance of the ETo methods: Hargreaves-Samani, Jensen-Haise, Benavides \& Lopez and Hamon using the standard method Penman Monteith FAO-56, to estimate the daily ETo of six regions, three of the state of Rio de Janeiro and three of the state of Espírito Santo. The meteorological variables employed were provided by the Center for Weather Forecasting and Climate Studies and the National Institute of Space Research - (CPTEC - INPE). The performance of the models was evaluated by different statistical techniques where the model that best suited the studied locations in the two states was the Jensen Haise, obtaining correlation indexes (r) between 0.73 and 0.94 and reliability (C) between 0.60 and 0.90 with the Penman Monteith model.
\end{abstract}

Keywords: irrigation, meteorological data, Penman Monteith.

Autor de correspondência: Eluã Ramos Coutinho, eluaramos@hotmail.com 


\section{Introdução}

É essencial conscientizar a população sobre uso racional dos recursos hídricos, expondo cada vez mais para a sociedade que os mesmos dependem de um período de regeneração e que o uso irresponsável pode causar a escassez. Tais fatores já se comprovam em determinadas regiões do Brasil, onde é possível observar que a estiagem de chuvas ocasionam os baixos níveis de rios e reservatórios, prejudicando a produção agrícola e causando problemas como restrições de abastecimento.

$\mathrm{Na}$ agricultura, a irrigação tem a finalidade de possibilitar a produção de culturas durante diferentes épocas do ano, sendo para determinadas regiões uma ferramenta indispensável, possibilitando rendimentos satisfatórios e mantendo a produção em locais onde seu cultivo normal seria inviável (Dantas et al., 2016). No entanto, a má utilização de água por meio deste processo é um fator preocupante na atualidade.

Desta forma, determinar a quantidade de água ideal para o desenvolvimento de uma determinada cultura é fundamental para dimensionar sistemas de irrigação e para o manejo da água na agricultura (Chagas et al., 2013).

Assim, é indispensável estimar de forma adequada a evapotranspiração da cultura (ETc), que é o principal parâmetro para o dimensionamento e manejo de sistemas de irrigação. A ETc representa a quantidade de água utilizada nos processos de evaporação e transpiração pela cultura, durante determinado período (Sousa et al., 2010).

A ETc é determinada a partir do produto da evapotranspiração de referência $(E T o)$ e o coeficiente da cultura $(\mathrm{Kc})$, que varia de acordo com o tipo de cultura e seu estágio fenelógico (Moura et al., 2013).

A ETo é uma variável relevante para o planejamento da irrigação, podendo ser obtida principalmente por meio de lisímetros, porém sua implantação é de alto custo, e para que haja precisão nas medições os mesmos devem ser manuseados por pessoas treinadas (Fernandes et al., 2012). Entretanto, sua estimativa pode ser realizada por diferentes métodos empíricos.

Segundo Sousa et al. (2010), visando uma padronização do calculo da $E T o$, a Food and Agriculture Organization (FAO) propôs o método de Penman-Monteith, com base em estudos de uma comissão de especialista em 1991, como modelo padrão para quantificar a demanda atmosférica de evapotranspiração.

Apesar do modelo de Penman-Monteith demonstrar resultados apurados na estimativa da ETo em diferentes condições climáticas, ele necessita de diversas variáveis meteorológicas, como radiação solar, temperatura do ar, umidade relativa do ar, velocidade do vento, entre outras, que nem sempre estão disponíveis em todas as regiões (Camargo e Camargo 2000, Santos et al., 2016). Para tanto, diferentes modelos que utilizam um menor número de variáveis tem sido comumente aplicados como alterna- tivas ao Penman-Monteith. Porém, esses modelos podem não apresentar boas estimativas, superestimando ou subestimando a ETo quando comparados com o método padrão (Dantas et al., 2016).

Mendonça et al. (2003), comparou os métodos de Penman-Monteith- FAO, Makkink, Linacre, Jensen-Haise, Hargreaves-Samani, Radiação Solar, Tanque Classe A, e Atmômetro SEEI modificado para região de Campos dos Goytacazes- RJ, a fim de propor coeficientes de ajuste regional entre os métodos empíricos e o método lisimétrico. Os resultados demonstraram que apenas o método do Atmômetro não atendeu satisfatoriamente a estimativa da ETo na região.

Dantas et al. (2016), comparou a precisão dos métodos de Hargreaves-Samani, Jensen-Haise, Blaney-Criddle e Camargo, em relação ao método de Penman-Monteith para mesorregiões do estado da Paraíba. A avaliação dos resultados indicou que o modelo de Hargreaves-Samani não necessitou de ajustes para região do agreste, mas ele não se ajustou à região de Borborema. Entretanto, os métodos de Jensen-Haise, Blaney-Criddle e Camargo, necessitaram de ajustes para as mesorregiões do Litoral, Sertão Paraibano e Borborema. Santos et al. (2017) comparou os métodos de Ivanov, Jensen-Haise, Makkink, PriestleyTaylor, Villa Nova, Hargreaves e Samani e Hamon com o método de Penman-Monteith para região de Petrolina PE. Os resultados demonstraram que o modelo que melhor se adaptou a região foi o modelo de Jensen-Haise.

Já Borges e Mediondo (2007), verificaram a precisão dos métodos de Camargo, Blaney-Criddle, Hamon, Hargreaves, Thornthwaite e Kharrufa, na estimativa de ETo da Bacia do rio Jacupiranga. Os resultados indicaram que os métodos de Hargreaves e Camargo foram mais eficientes na estimativa da ETo.

Dentro deste contexto, o objetivo do presente estudo foi avaliar e comparar o desempenho de métodos empíricos de estimativa da ETo, Hargreaves-Samani, JensenHaise, Linacre, Benavides \& Lopez e Hamon com o método padrão de Penman-Monteih, sugerido pela FAO56 , para 6 localidades pertencentes a dois estados da região sudeste do Brasil.

\section{Materiais e Métodos}

\subsection{Base de dados}

O presente estudo utilizou uma série de informações meteorológicas pertencentes a seis estações do Centro de Previsão de Tempo e Estudos Climáticos e do Instituto Nacional de Pesquisas Espaciais - (CPTEC - INPE), localizadas nos estados do Rio de Janeiro e Espírito Santo (Tabela 1).

Estas foram informações diárias calculadas a partir das variáveis horárias de temperatura média, máxima e mínima do ar $\left({ }^{\circ} \mathrm{C}\right)$, umidade relativa do ar $(\%)$, radiação 
Tabela 1 - Localização das estações utilizadas.

\begin{tabular}{lccc}
\hline Estações & Estado & Latitude & Longitude \\
\hline Mendes & RJ & $-22,521^{\circ} \mathrm{S}$ & $-43,721^{\circ} \mathrm{W}$ \\
Santa Maria Madalena & RJ & $-21,953^{\circ} \mathrm{S}$ & $-42,005^{\circ} \mathrm{W}$ \\
Teresópolis & RJ & $-22,407^{\circ} \mathrm{S}$ & 615 \\
Jerônimo Monteiro & ES & $-20,750^{\circ} \mathrm{S}$ & $-42,793^{\circ} \mathrm{W}$ \\
Sooretama & ES & $-19,367^{\circ} \mathrm{S}$ & $-41,288^{\circ} \mathrm{W}$ \\
Venda Nova Imigrante & ES & $-20,384^{\circ} \mathrm{S}$ & $-40,074^{\circ} \mathrm{W}$ \\
\hline
\end{tabular}

solar global ( $\left.\mathrm{MJ} \mathrm{m}^{-2} \mathrm{~d}^{-1}\right)$ e velocidade do vento medida a 10 metros de altura $\left(\mathrm{m} \mathrm{s}^{-1}\right)$ e corrigida segundo Bezerra et al. (2009), por meio da Eq. (1), registradas no período de 01/01/2005 a 31/12/2015.

$$
u_{2}=u_{z} \frac{4,87}{\ln (67,8 z-5,42)}
$$

onde $u_{2}$ - velocidade do vento a $2 \mathrm{~m}$ de altura $\left(\mathrm{m} \mathrm{s}^{-1}\right) ; u_{z}$ velocidade do vento a altura $z\left(\mathrm{~m} \mathrm{~s}^{-1}\right) ; \mathrm{Z}$ - altura onde a velocidade do vento está sendo medida (m).

\subsection{Método de Penman-Monteith}

Este método foi desenvolvido por Penman-Monteith, devido a sua consistência para estimar a ETo em diferentes locais e climas e ainda considerar parâmetros de trocas de energia e fluxo de calor latente, ele foi definido em 1990 pela FAO como método padrão para estimativa de ETo (Vescove e Turco, 2005; Cardoso e Justino, 2014). O método do Penman-Monteith estima a ETo em $\mathrm{mm} \mathrm{d}^{-1}$ Eq. (2).

$$
\text { ETo }=\frac{0,408 \Delta\left(R_{n}-G\right)+\gamma\left(\frac{900}{T_{a}+273}\right) u_{2}\left(e_{s}-e_{a}\right)}{\Delta+\gamma\left(1+0,34 u_{2}\right)}
$$

onde $E T o$ - evapotranspiração de referência, $\mathrm{mm} \mathrm{d}^{-1} ; \Delta$ curva de pressão de vapor, $\mathrm{kPa}{ }^{\circ} \mathrm{C}^{-1} ; R_{n}$ - saldo da radiação diário, $\mathrm{MJ} \mathrm{m}^{-2} \mathrm{~d}^{-1} ; G$ - fluxo de calor no solo, $\mathrm{MJ}$ $\mathrm{m}^{-2} \mathrm{~d}^{-1} ; \gamma$ - constante psicrométrica, $\mathrm{kPa}{ }^{\circ} \mathrm{C}^{-1} ; u_{2}$ - velocidade do vento a $2 \mathrm{~m}, \mathrm{~m} \mathrm{~s}^{-1} ; E_{s}$ - pressão de saturação do vapor de água atmosférico, $\mathrm{kPa} ; E_{a}$ - pressão atual do vapor de água atmosférico, $\mathrm{kPa} ; T_{a}$ - temperatura média do ar, ${ }^{\circ} \mathrm{C}$.

\subsection{Método de Hargreaves-Samani}

O método Hargreaves-Samani também estima a ETo $\mathrm{em} \mathrm{mm} \mathrm{d}^{-1}$. Ele pode ser utilizado na ausência dos dados de radiação solar, umidade relativa do ar e velocidade do vento, e é expresso pela Eq. (3) (Silva et al., 2005):

$$
\begin{array}{rl}
E T o=0.408 * & 0,0023\left(T_{\text {med }}+17,8\right) \\
& \times\left(T_{\max }-T_{\min }\right)^{0,5} R_{a}
\end{array}
$$

onde ETo - evapotranspiração de referência, $\mathrm{mm} \mathrm{d}^{-1} ; T_{\text {med }}$ - temperatura média do ar em ${ }^{\circ} \mathrm{C} ; T_{\max }$ - temperatura má- xima do ar em ${ }^{\circ} \mathrm{C} ; T_{\min }$ - temperatura mínima do ar em ${ }^{\circ} \mathrm{C}$; $R_{a}$ - radiação extraterrestre em $\mathrm{MJ} \mathrm{m}^{-2} \mathrm{~d}^{-1}$.

\subsection{Método de Jensen-Haise}

O método Jensen-Haise foi criado como uma alternativa ao Penman-Monteith para o cálculo da ETo, e pode ser aplicado em regiões áridas e semi-áridas por meio da Eq. (4) (Jensen et al., 1997).

$$
E T o=R_{s}\left(0,025 T_{a}+0,08\right)
$$

onde ETo - evapotranspiração de referência, $\mathrm{mm} \mathrm{d}^{-1} ; T_{a}$ temperatura média do ar diária em ${ }^{\circ} \mathrm{C} ; R_{S}$ - radiação solar global convertida em $\mathrm{mm}$.

\subsection{Método de Linacre}

O método de Linacre também estima a evapotranspiração em $\mathrm{mm} \mathrm{d}^{-1}$. Pode ser obtida em função da altitude, latitude e das temperaturas diárias máxima, mínima e do ponto de orvalho, através da Eq. (5) (Linacre, 1977).

$$
\text { ETo }=\frac{700 \frac{T_{m}}{100-\varphi}+15\left(T_{a}-T_{d}\right)}{\left(80-T_{a}\right)}
$$

onde $E T o$ - evapotranspiração de referência, $\mathrm{mm} \mathrm{d}^{-1} ; T_{m}$ $T_{a}+0,006 \mathrm{z}, \mathrm{z}$ é a altitude $(\mathrm{m})$ temperatura média do ar em ${ }^{\circ} \mathrm{C}$; $\varphi$ - latitude (graus); $T_{d}$ - temperatura média do ponto de orvalho.

\subsection{Método de Benavides \& Lopez}

Segundo Fanaya Júnior et al. (2012), este método foi desenvolvido por Garcia Benavides e Lopez Dias em 1970, e se baseia apenas em dados de temperatura média do ar e umidade relativa do ar, sendo a ETo estimada por meio da Eq. (6) (Matos e Silva, 2016).

$$
\begin{gathered}
\text { ETo }=1,21 * 10\left(\frac{7,45 T_{\text {med }}}{234,7+T_{\text {med }}}\right)\left(1-0,01 U R_{\text {med }}\right) \\
+0,21 T_{\text {med }}-2,30
\end{gathered}
$$

onde ETo - evapotranspiração de referência, $\mathrm{mm} \mathrm{d}^{-1} ; T_{\text {med }}$ - Temperatura média do ar ${ }^{\circ} \mathrm{C} ; U R_{\text {med }}$ - Média da umidade relativa do ar em $\%$. 


\subsection{Método de Hamon}

Este método utiliza a temperatura média do ar e a insolação máxima diária para estimar a evapotranspiração em $\mathrm{mm} \mathrm{d}^{-1}$, e é expresso pela Eq. (7) (Hamon, 1961).

$$
\text { ETo }=0,55\left(\frac{\mathrm{N}}{12}\right)^{2}\left(\frac{4,95 \exp ^{0,062 \mathrm{~T}_{\mathrm{a}}}}{100}\right) 25,4
$$

onde $E T o$ - evapotranspiração de referência, $\mathrm{mm} \mathrm{d}^{-1} ; N$ insolação máxima diária teórica, função da latitude e época do ano, $\mathrm{h} ; T_{a}$ - temperatura média do ar diária em ${ }^{\circ} \mathrm{C}$.

\subsection{Avaliação de desempenho}

Para comparar o desempenho dos modelos com os resultados apresentados pelo Penman-Monteith na estimativa da ETo foram utilizadas diferentes medidas estatísticas, como o coeficiente de correlação de Pearson $(r)$ Eq. (8), o erro médio absoluto (EMA) Eq. (9), raiz do erro médio quadrático $(R E M Q)$ Eq. (10), erro médio percentual $(E M P)$ Eq. (11), índice de concordância $(D)$ Eq. (12) e o índice de confiança (C) Eq. (13) (Fonseca et al., 2012, Pezzopane et al., 2012).

$$
\begin{gathered}
r=\frac{\frac{\sum_{j=1}^{N}\left(x_{j}-\bar{x}\right)\left(o_{j}-\bar{o}\right)}{N}}{\sqrt{\frac{\sum_{j=1}^{N}\left(x_{j}-\bar{x}\right)^{2}}{N}} \cdot \sqrt{\frac{\sum_{j=1}^{N}\left(o_{j}-\bar{o}\right)^{2}}{N}}} \\
E M A=\frac{\sum_{j=1}^{n}\left|o_{j}-x_{j}\right|}{n} \\
R E M Q=\sqrt{\frac{\sum_{j=1}^{n}\left(o_{j}-x_{j}\right)^{2}}{n}} \\
E M P=\frac{\sum_{j=1}^{n} \frac{\left|o_{j}-x_{j}\right|}{o_{j}}}{n} * 100
\end{gathered}
$$

O índice de confiança $(C)$ permite analisar conjuntamente a precisão e a exatidão dos resultados obtidos. Ele é calculado através do produto do coeficiente de correlação $(r)$ pelo índice de concordância $(D)$. Seus valores variam de zero (0) para nenhuma concordância a um (1) para a concordância perfeita (Pezzopane et al., 2012). A Tabela 2 mostra os critérios para avaliação de desempenho.

$$
\begin{gathered}
D=1-\frac{\sum_{j=1}^{n}\left(o_{j}-x_{j}\right)^{2}}{\sum_{j=1}^{n}\left(\left|x_{j}-\bar{o}\right|+\left|o_{j}-\bar{o}\right|\right)^{2}} \\
C=(r * D)
\end{gathered}
$$

onde $n$ ou $N$ representa o número de dados utilizados, $O_{j}$ o valor observado, $x_{j}$ o valor estimado pelas técnicas empre-
Tabela 2 - Critérios para avaliação e análise do desempenho de modelos com base no índice de confiança

\begin{tabular}{lc}
\hline Valor de IC & Desempenho \\
\hline$>0,85$ & Ótimo \\
0,76 a 0,85 & Muito bom \\
0,66 a 0,75 & Bom \\
0,61 a 0,65 & Mediano \\
0,51 a 0,60 & Sofrível \\
0,41 a 0,50 & Mau \\
$\leq 0,40$ & Péssimo \\
\hline
\end{tabular}

gadas, $\bar{O}$ a média dos dados observados e $\bar{x}$ a média dos dados estimados.

Além destes métodos, também foram utilizados para comparar a ETo estimada com Penman-Monteith com a ETo estimada pelos demais modelos, as medidas estatísticas de média (Med), máxima (Max), mínima (Min) e desvio padrão (Desv Pad).

\section{Resultados e Discussão}

\subsection{Resultados apresentados na estimativa da ETo do estado do Rio de Janeiro}

Analisando os resultados destacados pelas medidas de (r), REMQ, EMA, $(D)$ e $(C)$ na Tabela 3, nota-se que em duas das três regiões do estado do Rio de Janeiro, o modelo de Jensen Haise foi superior em suas estimativas. Tal fato pode ser confirmado comparando os resultados da medida de erro (MPE) obtida pelo modelo de Jensen Haise em suas estimativas, onde a mesma foi consideravelmente a menor em todas as regiões do estado, mantendo-se entre $44 \%$ a $160 \%$ mais precisa que os demais modelos.

Comparando todos os resultados dos modelos para estimativas de $E T o$, observa-se que para região de Mendes, além do modelo de Jensen Haise, os modelos de Linacre e Benavides Lopez também demonstravam os maiores índices de $(r)$ com o modelo de Penman Monteith. Porém, suas medidas de REMQ, EMA e EMP indicavam que a precisão dos resultados expostos por estes modelos era baixa, sendo inferiores a todos os outros.

Já na região de Santa Maria Madalena, o modelo que apresentou os maiores índices de $(r)$ foi o Benavides Lopez, tendo também demonstrado os maiores $(D)$ e $(C)$. Contudo, o erro $R E M Q$ foi superior ao apresentado pelo modelo Linacre, e o EMA e o EMP foram maiores que o do Jensen Haise, o que caracteriza que apesar de sua melhor adaptação nesta região, seus valores estimados apresentavam maior variação.

Para Teresópolis, também comprova-se que a ETo estimada pelo Jensen Haise alcançou os maiores valores de $(r),(D)$ e $(C)$, o que demonstra uma alta relação com a ETo estimada com o Penman Monteith. Outro ponto de destaque que se observa por meio da Tabela 3 é que as 
Tabela 3 - Comparação entre a ETo estimada com Penman Monteith e outros modelos para o estado do Rio de Janeiro. Índices: média ( $M e d)$, máxima $(M a x)$, mínima $(M i n)$, desvio padrão (Desv Pad), coeficiente de correlação $(r)$, raiz do erro médio quadrático $(R E M Q)$, erro médio absoluto $(E M A)$, erro médio percentual $(E M P)$, índice de concordância $(D)$, índice de confiança $(C)$. Modelos: Hargreaves Samani, Hamon, Jensen Haise, Linacre, Benavides Lopez.

\begin{tabular}{|c|c|c|c|c|c|c|c|c|c|c|c|}
\hline \multicolumn{4}{|c|}{ Penman-Monteith - ETo - RJ } & \multicolumn{2}{|c|}{ Med } & \multicolumn{2}{|c|}{ Max } & \multicolumn{2}{|c|}{ Min } & \multicolumn{2}{|c|}{ Desv pad } \\
\hline Mendes & & & & \multicolumn{2}{|r|}{3,49} & \multicolumn{2}{|c|}{8,97} & \multicolumn{2}{|c|}{0,45} & \multicolumn{2}{|c|}{1,70} \\
\hline \multicolumn{4}{|l|}{ Santa Maria Madalena } & \multicolumn{2}{|c|}{3,96} & \multicolumn{2}{|c|}{10,80} & \multicolumn{2}{|c|}{0,45} & \multicolumn{2}{|c|}{1,78} \\
\hline \multicolumn{2}{|l|}{ Teresópolis } & & & \multicolumn{2}{|r|}{3,83} & \multicolumn{2}{|c|}{9,86} & \multicolumn{2}{|c|}{0,45} & \multicolumn{2}{|c|}{1,68} \\
\hline ETo- Estimada & Modelos & Med & $\operatorname{Max}$ & Min & Desv pad & (r) & REMQ & EMA & EMP (\%) & (D) & (C) \\
\hline \multirow{5}{*}{ Mendes } & Hargreaves-Samani & 4,45 & 8,64 & 1,01 & 1,40 & 0,67 & 1,49 & 1,17 & 59,12 & 0,74 & 0,50 \\
\hline & Hamon & 3,41 & 7,69 & 1,27 & 1,14 & 0,66 & 1,27 & 1,01 & 45,16 & 0,76 & 0,50 \\
\hline & Jensen-Haise & 4,35 & 13,38 & 0,02 & 2,56 & 0,93 & 1,42 & 1,04 & 28,36 & 0,89 & 0,83 \\
\hline & Linacre & 4,78 & 11,61 & 1,62 & 1,45 & 0,78 & 1,67 & 1,43 & 66,45 & 0,73 & 0,57 \\
\hline & Benavides-Lopez & 5,26 & 12,89 & 0,58 & 1,98 & 0,79 & 2,14 & 1,85 & 72,27 & 0,70 & 0,55 \\
\hline \multirow[t]{3}{*}{ ETo- Estimada } & Modelos & Med & $\operatorname{Max}$ & Min & Desv pad & (r) & REMQ & EMA & EMP (\%) & (D) & (C) \\
\hline & Hargreaves-Samani & 4,15 & 7,69 & 0,61 & 1,31 & 0,71 & 1,25 & 0,97 & 36,92 & 0,80 & 0,57 \\
\hline & Hamon & 2,93 & 5,74 & 1,28 & 0,89 & 0,67 & 1,70 & 1,36 & 36,58 & 0,59 & 0,39 \\
\hline \multirow[t]{3}{*}{ Santa Maria Madalena } & Jensen-Haise & 3,63 & 11 & 0,01 & 2,15 & 0,81 & 1,30 & 0,87 & 22,88 & 0,87 & 0,71 \\
\hline & Linacre & 4,58 & 10,55 & 1,78 & 1,32 & 0,86 & 1,11 & 0,90 & 35,56 & 0,86 & 0,74 \\
\hline & Benavides-Lopez & 4,83 & 10,97 & 0,71 & 1,72 & 0,89 & 1,18 & 0,94 & 32,88 & 0,88 & 0,79 \\
\hline \multirow[t]{3}{*}{ ETo- Estimada } & Modelos & Med & $\operatorname{Max}$ & Min & Desv pad & (r) & REMQ & EMA & $\operatorname{EMP}(\%)$ & (D) & (C) \\
\hline & Hargreaves-Samani & 4,37 & 8,33 & 1,13 & 1,40 & 0,76 & 1,21 & 0,92 & 35,30 & 0,83 & 0,63 \\
\hline & Hamon & 2,95 & 6,27 & 1,09 & 0,97 & 0,71 & 1,47 & 1,18 & 31,88 & 0,68 & 0,48 \\
\hline \multirow[t]{3}{*}{ Teresópolis } & Jensen-Haise & 3,69 & 9,78 & 0,02 & 2,03 & 0,90 & 0,90 & 0,68 & 19,54 & 0,93 & 0,84 \\
\hline & Linacre & 4,73 & 12,03 & 1,89 & 1,30 & 0,85 & 1,25 & 1,07 & 40,53 & 0,82 & 0,70 \\
\hline & Benavides-Lopez & 4,84 & 12,82 & 0,58 & 1,79 & 0,85 & 1,37 & 1,12 & 36,78 & 0,84 & 0,72 \\
\hline
\end{tabular}

medidas de erros REMQ, EMA e EMP geradas com o modelo de Jensen Haise foram inferiores aos erros exibidos pelos demais modelos, mantendo-se em 0,91 para $R E M Q, 0,68$ para $E M A$ e $19,56 \%$ para $E M P$. A comparação entre o melhor e o pior resultado exposto pelos modelos em relação ao método padrão (Penman Monteith) pode ser verificado na Fig. 1.

\subsection{Resultados apresentados na estimativa da ETo do estado do Espírito Santo}

É possível observar a partir da Tabela 4 que o modelo de Jensen Haise demonstrou maior aptidão nas regiões de Jerônimo Monteiro e Venda Nova do Imigrante, onde seus índices de $(r)$ expõem uma alta relação com a ETo estimada pelo Penman Monteith. Porém, para a região de Sooretama o modelo de Linacre apresenta o maior índice de $(r)$, o que garante a maior relação com o modelo de Penman Monteith.
Analisando todos os modelos na estimativa de ETo, é possível observar a partir de seus resultados que para a região de Jerônimo Monteiro o modelo de Jensen Haise também apresenta os menores valores de REMQ, EMA, $(D)$ e $(C)$, e que seu $E M P$ é duas vezes menor que o obtido pelo modelo de Linacre. Contudo, o modelo Linacre também apresenta altos valores de $(r),(D)$ e $(C)$ o que determina uma alta associação com os valores do Penman Monteith e o que o caracterizaria como a segunda melhor alternativa na estimativa da ETo para essa região.

Na região de Sooretama, o modelo de Linacre apresenta os menores valores de REMQ e EMA e também o maior índice $(C)$. No entanto, o erro EMP apresentado pelo modelo Linacre é superior ao obtido pelo modelo de Jensen Haise, o que indica que independentemente de sua melhor adaptação, o mesmo apresenta uma maior variação na estimativa da ETo em comparação com o modelo de Jensen Haise. 

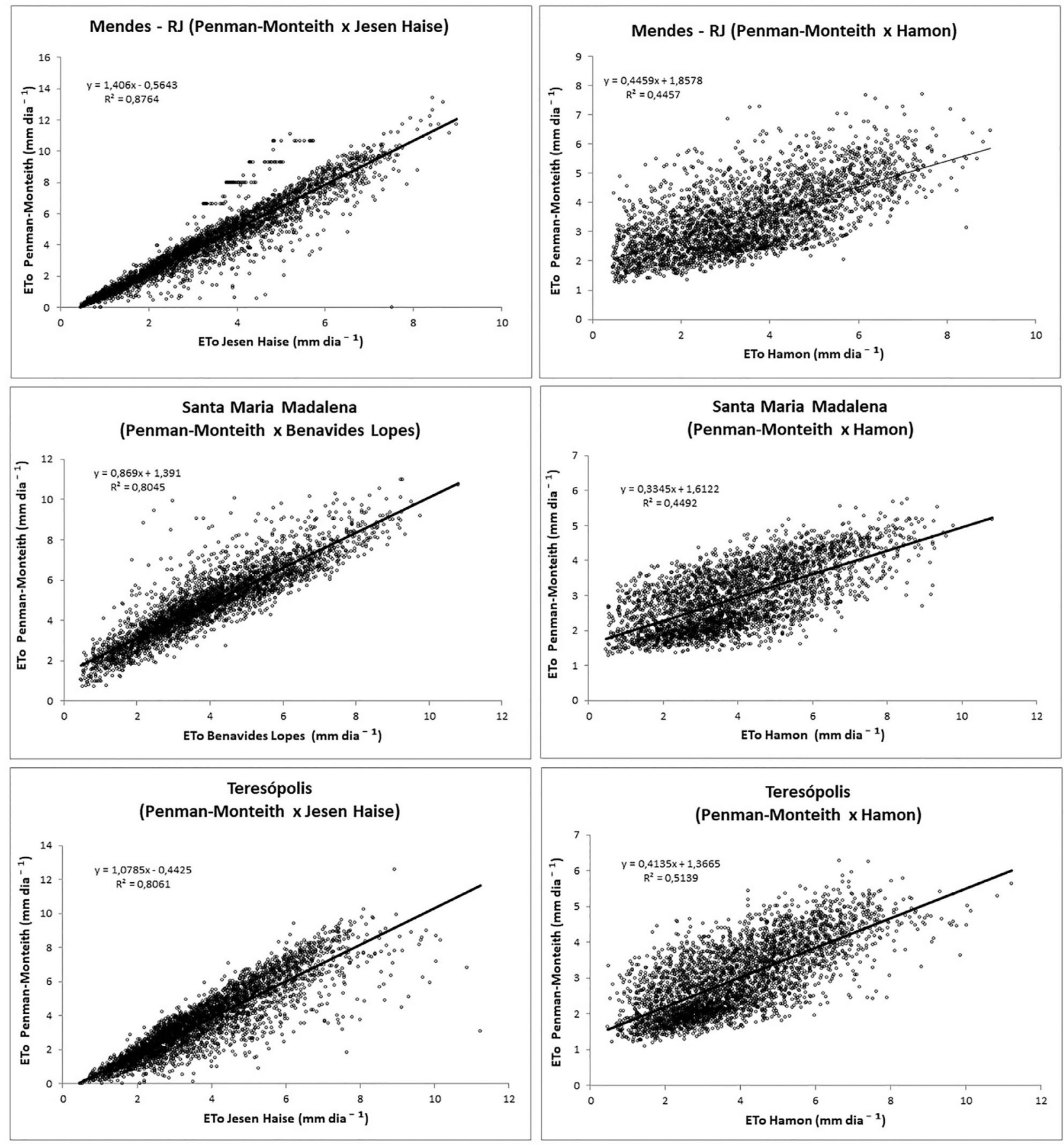

Figura 1 - Gráficos de dispersão dos melhores e piores resultados apresentados pelos modelos para as regiões do estado do Rio de Janeiro.

Para Venda Nova do Imigrante, verifica-se que a ETo estimada pelo Jensen Haise também exibiu uma alta relação com os valores do modelo de Penman Monteith, onde o modelo de Jensen Haise alcançou os maiores valores de $(r),(D)$ e $(C)$. Outro fator relevante que pode ser observado a partir da Tabela 4, é que as medidas de erros $R E M Q$, EMA e EMP geradas com o modelo de
Jensen Haise foram consideravelmente menores que os erros exibidos pelos demais modelos, mantendo-se em 0,83 para $R E M Q, 0,62$ para $E M A$ e $18,61 \%$ para $E M P$, o que é quase três vezes menor que o $E M P$ obtido pelo Benavides Lopez. A comparação entre o melhor e o pior resultado exposto pelos modelos pode ser verificado na Fig. 2. 
Tabela 4 - Comparação entre a ETo estimada com Penman Monteith e outros modelos para o estado do Espírito Santo. Índices: média (Med), máxima $(M a x)$, mínima (Min), desvio padrão (Desv Pad), coeficiente de correlação $(r)$, raiz do erro médio quadrático $(R E M Q)$, erro médio absoluto $(E M A)$, erro médio percentual $(E M P)$, índice de concordância $(D)$, índice de confiança $(C)$. Modelos: Hargreaves Samani, Hamon, Jensen Haise, Linacre, Benavides Lopez.

\begin{tabular}{|c|c|c|c|c|c|c|c|c|c|c|c|}
\hline \multicolumn{4}{|c|}{ Penman-Monteith - ETo - ES } & \multicolumn{2}{|r|}{ Med } & \multicolumn{2}{|c|}{$\operatorname{Max}$} & \multicolumn{2}{|r|}{ Min } & \multicolumn{2}{|c|}{ Desv Pad } \\
\hline Jerônimo Monteiro & & & & \multicolumn{2}{|r|}{4,51} & \multicolumn{2}{|c|}{10,76} & \multicolumn{2}{|r|}{0,50} & \multicolumn{2}{|c|}{2,12} \\
\hline \multicolumn{4}{|l|}{ Sooretama } & \multicolumn{2}{|r|}{4,60} & \multicolumn{2}{|c|}{10,00} & \multicolumn{2}{|r|}{0,47} & \multicolumn{2}{|c|}{1,76} \\
\hline \multicolumn{4}{|l|}{ Venda Nova do Imigrante } & \multicolumn{2}{|r|}{3,60} & \multicolumn{2}{|c|}{9,32} & \multicolumn{2}{|r|}{0,49} & \multicolumn{2}{|c|}{1,83} \\
\hline ETo- Estimada & Modelos & Med & $\operatorname{Max}$ & Min & Desv pad & (r) & REMQ & EMA & $\operatorname{EMP}(\%)$ & (D) & (C) \\
\hline \multirow{5}{*}{ Jerônimo Monteiro } & Hargreaves-Samani & 5,09 & 8,70 & 1,53 & 1,47 & 0,73 & 1,55 & 1,16 & 49,37 & 0,79 & 0,58 \\
\hline & Hamon & 3,94 & 9,13 & 1,55 & 1,22 & 0,73 & 1,58 & 1,29 & 39,16 & 0,75 & 0,55 \\
\hline & Jensen-Haise & 5,02 & 12,79 & 0,02 & 2,85 & 0,93 & 1,26 & 0,97 & 22,65 & 0,93 & 0,87 \\
\hline & Linacre & 5,53 & 11,70 & 2,05 & 1,68 & 0,86 & 1,48 & 1,25 & 45,76 & 0,84 & 0,73 \\
\hline & Benavides-Lopez & 6,68 & 13,34 & 1,67 & 2,17 & 0,87 & 2,42 & 2,18 & 68,28 & 0,73 & 0,63 \\
\hline \multirow[t]{3}{*}{ ETo- Estimada } & Modelos & Med & Max & Min & Desv pad & (r) & REMQ & EMA & EMP (\%) & (D) & (C) \\
\hline & Hargreaves-Samani & 4,69 & 8,82 & 1,45 & 1,21 & 0,69 & 1,28 & 0,97 & 30,30 & 0,78 & 0,54 \\
\hline & Hamon & 3,65 & 9,02 & 1,73 & 0,94 & 0,70 & 1,63 & 1,33 & 31,03 & 0,63 & 0,44 \\
\hline \multirow[t]{3}{*}{ Sooretama } & Jensen-Haise & 4,46 & 11,60 & 0,01 & 2,40 & 0,74 & 1,56 & 1,16 & 25,69 & 0,83 & 0,62 \\
\hline & Linacre & 5,07 & 12,15 & 1,66 & 1,17 & 0,77 & 1,21 & 0,98 & 29,91 & 0,81 & 0,63 \\
\hline & Benavides-Lopez & 6,24 & 13,69 & 1,12 & 1,54 & 0,82 & 1,92 & 1,65 & 48,64 & 0,71 & 0,58 \\
\hline \multirow[t]{3}{*}{ ETo- Estimada } & Modelos & Med & $\operatorname{Max}$ & Min & Desv pad & (r) & REMQ & EMA & EMP (\%) & (D) & (C) \\
\hline & Hargreaves-Samani & 4,47 & 8,54 & 1,13 & 1,38 & 0,79 & 1,42 & 1,12 & 55,21 & 0,79 & 0,63 \\
\hline & Hamon & 3,03 & 9,07 & 1,27 & 0,97 & 0,69 & 1,46 & 1,17 & 40,43 & 0,69 & 0,48 \\
\hline \multirow[t]{3}{*}{ Venda Nova do Imigrante } & Jensen-Haise & 3,81 & 12,61 & 0,01 & 2,26 & 0,94 & 0,83 & 0,62 & 18,61 & 0,95 & 0,90 \\
\hline & Linacre & 4,62 & 12,81 & 1,82 & 1,38 & 0,87 & 1,37 & 1,16 & 54,56 & 0,82 & 0,72 \\
\hline & Benavides-Lopez & 4,93 & 13,05 & 0,73 & 1,91 & 0,88 & 1,59 & 1,35 & 51,70 & 0,82 & 0,73 \\
\hline
\end{tabular}

\section{Conclusões}

A partir da análise dos resultados, é possível concluir que o modelo que melhor se adaptou às localidades estudadas nos dois estados foi o de Jensen Haise, tendo obtido na maioria das regiões os maiores índices de correlação $(r)$ e confiabilidade $(C)$ com o modelo de Penman Monteith.

Os modelos de Hargreaves Samani e Hamon demonstraram os menores índices de $(r)$ e $(C)$, caracterizando uma baixa eficiência na estimativa da ETo nas localidades estudadas.
Também pode ser obervado que apesar dos modelos de Linacre e Benavides Lopez terem apresentado altos índices de $(r)$ e $(C)$, os mesmos tiveram altas variações em suas estimativas, constatadas por meio dos índices de $(R E M Q),(E M A)$ e $(E M P)$.

Contudo, ainda que seja possível observar melhor adaptação do modelo de Jensen Haise na estimativa da ETo, seus resultados não foram predominantes para todas as regiões. Assim, a avaliação de outros modelos pode apresentar resultados mais apropriados para as mesmas. Entretanto, pode-se afirmar que o modelo de Jensen Haise é uma alternativa viável para estimar a ETo nestas localidades. 

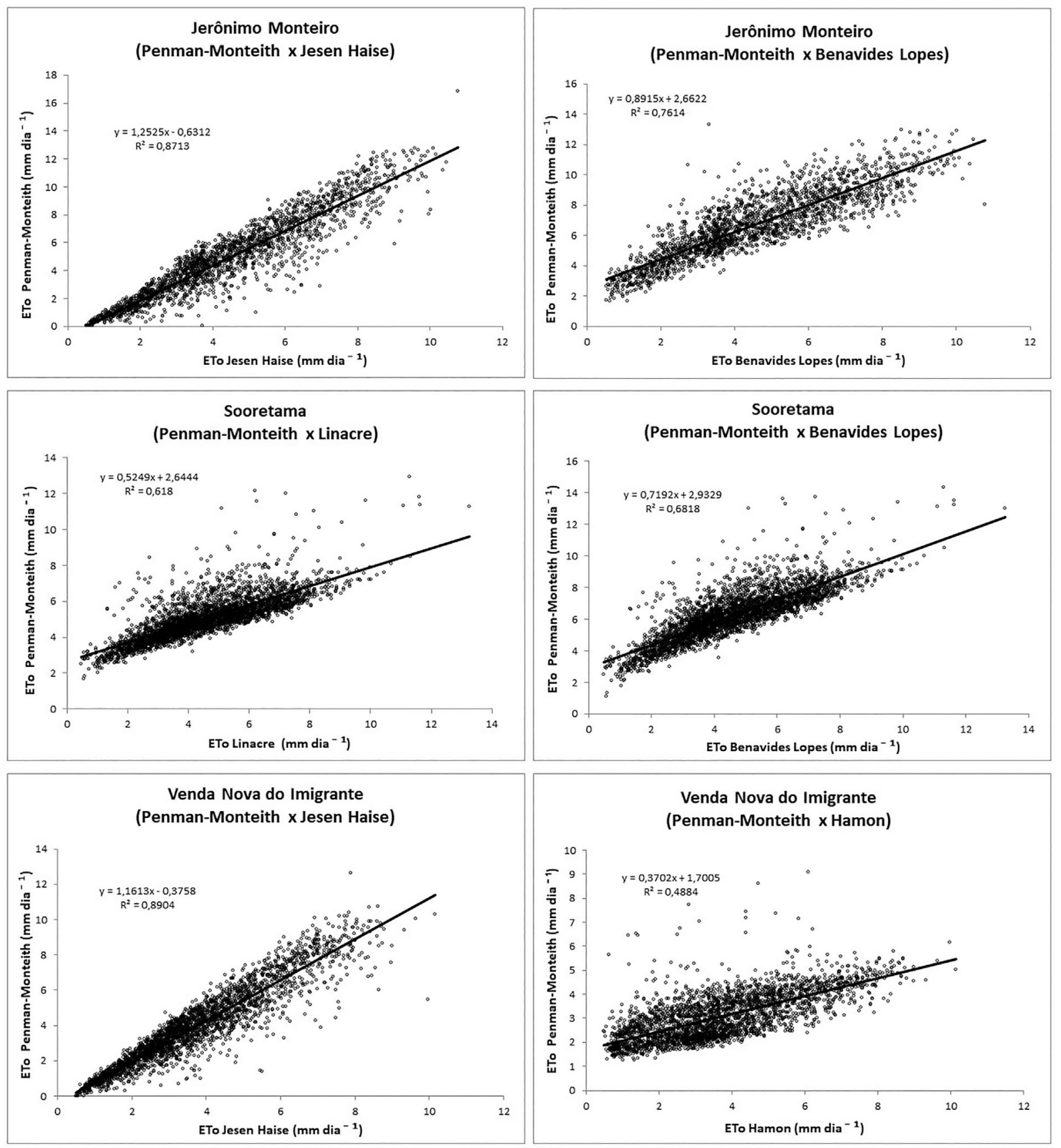

Figura 2 - Gráficos de dispersão dos melhores e piores resultados apresentados pelos modelos para as regiões do estado do Espírito Santo.

\section{Agradecimentos}

Agradecemos a Coordenação de Aperfeiçoamento de Pessoal de Nível Superior (CAPES) pelo fornecimento da bolsa de pesquisa, e ao Centro de Previsão de Tempo e Estudos Climáticos, Instituto Nacional de Pesquisas Espaciais - (CPTEC - INPE) por disponibilizar os dados meteorológicos, o Centro federal de educação Tecnológica Celso Suckow da Fonseca (CEFET-ANGRA) e a Uni- versidade Federal Rural do Rio de Janeiro (UFRRJ-SEROPÉDICA).

\section{Referências}

BEZERRA, J.R.C.; DIAS, J.M.; PEREIRA, J.R.; GUEDES, F.X. Consumo hídrico do algodoeiro BRS 200 Marrom. Circular Técnica 129-EMBRAPA. Campina Grande: Embrapa Algodão, p. 11, 2009. 
BORGES, A.C.; MENDIONDO, E.M. Comparação entre equações empíricas para estimativa da evapotranspiração de referência na bacia do rio Jacupiranga. Revista Brasileira de Engenharia Agrícola e Ambiental, v. 11, n. 3, p. 293300, 2007.

CAMARGO, A.P.; CAMARGO, M.B.P. Uma revisão analítica da evapotranspiração potencial. Bragantina, Campinas, v. 59, n. 2 , p. $125-137,2000$.

CARDOSO, G.M.; JUSTINO, F. Simulação dos componentes da evapotranspiração sob condições climáticas atuais e de cenários climáticos futuros de aquecimento global com o uso de modelos de clima-vegetação. Revista Brasileira de Meteorologia, v. 29, n. 1, p. 85-95, 2014.

CHAGAS, R.M.; FACCIOLI, G.G.; AGUIAR NETTO, A.O.; SOUSA, I.F.; VASCO, A.N.; SILVA, M.G. G. Comparação entre métodos de estimativa da evapotranspiração de referência (ETo) no município de Rio Real-Ba. Irriga, v. 18, n. 1, p. 351-363, 2013.

DANTAS, G.F.; OLIVEIRA, V.M.R.; DALRI, A.B.; PALARETTI, L.F.; SANTOS, M.G.; FARIA, R.T. T. Desempenho de métodos na estimativa de evapotranspiração de referência para o estado da Paraíba, Brasil. Irriga, v. 21, n. 3, p. 481-490, 2016.

FANAYA JÚNIOR, E.D.; LOPES, A.S.; OLIVEIRA, G.Q.; JUNG, L.H. H. Métodos empíricos para estimativa da evapotranspiração de referência para a Aquidauana, MS. Irriga, v. 17, n. 4, p. 418-438, 2012.

FERNANDES, L.C.; PAIVA, C.M.; ROTUNNO FILHO, O.C. Evaluation of six empirical evapotranspiration equations case study: Campos dos Goytacazes/Rj. Revista Brasileira de Meteorologia, v. 27, n. 3, p. 272-280, 2012.

FONSECA, J.S.; MARTINS, G.A.; TOLEDO, G.L. Estatística Aplicada. São Paulo: Atlas, Ed. 2, p. 267, 2012.

HAMON, W.R. Estimating potential evapotranspiration. Journal of Hydraulics Division, v. 87, n. 3, p. 107-120, 1961.

JENSEN, D.T.; HARGREAVES, G.H.; TEMESGEN, B. Computation of ETo under nonideal conditions. Journal or Irrigation and Drainage Engineering, v. 123, n. 5, p. 394-399, 1997.

LINACRE, E.T. A simple formula for estimating evaporation rates in various climates, using temperature data alone. Agricultural Meteorology, v. 18, n. 6, p. 409-424, 1977.

MATOS, R.M.; SILVA, P.F.; Análise de métodos de estimativa da evapotranspiração de referência mensal para o municí- pio de Barbalha-CE. E. Agropecuária Científica no Semiárido-ACSA, v. 12, n. 1, p. 10-21, 2016.

MENDONÇA, J.C.; SOUSA, E.F.; BERNARDO, S.; DIAS, G.P.; GRIPPA, S. S. comparação entre métodos de estimativa da evapotranspiração de referência $(E T o)$ na região Norte Fluminense, RJ. Revista Brasileira de Engenharia Agrícola e Ambiental, v. 7, n. 2, p. 275-279, 2003.

MOURA, A.R.C.; MONTENEGRO, S.M.G.L.; ANTONINO, A.C.D.; AZEVEDO, J.R.G.; SILVA, B.B.; OLIVEIRA, L.M.M. M. Evapotranspiração de referência baseada em métodos empíricos em bacia experimental no estado de Pernambuco - Brasil. Revista Brasileira de Meteorologia, v. 28, n. 2, p.181-191, 2013.

PEZZOPANE, J.E.M.; CASTRO, F.S.; PEZZOPANE, J.R.M.; CECÍLIO, R.A. A. Agrometeorologia Aplicações para o Espírito Santo, Alegre: CAUFES, p.163, 2012.

SANTOS, A.A.R.; LYRA, G.B.; LYRA, G.B.; LIMA, E.P.; SOUZA, J.L.; DELGADO, R.C. C. Evapotranspiração de referência em função dos extremos da temperatura do ar no estado do Rio de Janeiro. Irriga, v. 21, n. 3, p. 449-465, 2016.

SANTOS, R.D.S.; SOUZA, M.H.C.; BISPO, R.C.; VENTURA, K.M.; BASSOI, L.H. H. Comparação entre métodos de estimativa da evapotranspiração de referência para o município de Petrolina, PE. Irriga, v.1, n.1, p. 31-39, 2017.

SILVA, V.P.R.; BELO FILHO, A.F.; SILVA, B.B.; CAMPOS, J.H.B.C. C. Desenvolvimento de um sistema de estimativa da evapotranspiração de referência. Revista Brasileira de Engenharia Agrícola e Ambiental, v. 9, n. 4, p. 547-553, 2005.

SOUSA, I.F.; SILVA, V.P.R.; SABINO, F.G.; NETTO, A.O.A.; SILVA, B.K.N.; AZEVEDO, P.V. V. Evapotranspiração de referência nos perímetros irrigados do estado de Sergipe. Revista Brasileira de Engenharia Agrícola e Ambiental, v. 14, n. 6, p. 633-644, 2010.

VESCOVE, H.V.; TURCO, J.E.P. Comparação de três métodos de estimativa da evapotranspiração de referência para a região de Araraquara - SP. Associação Brasileira de Engenharia Agrícola, v. 25, n. 3, p. 713-721, 2005.

License information: This is an open-access article distributed under the terms of the Creative Commons Attribution License (type CC-BY), which permits unrestricted use, distribution and reproduction in any medium, provided the original article is properly cited. 